\section{THU0034 THE INFLAMMATORY POTENTIAL OF CALCIUM PYROPHOSPHATE CRYSTALS DEPENDS ON THEIR CAPACITY TO INDUCE NF-KB AND MAPK PATHWAYS}

F. Renaudin ${ }^{1,2}$, L. Campillo-Gimenez ${ }^{1}$, P. Gras ${ }^{3}$, C. Combes ${ }^{3}$, M. Cohen-Solal ${ }^{1,4}$, F. Lioté ${ }^{1,4}$, H.-K. Ea ${ }^{1,4}$. ${ }^{1}$ UMR1132, Bioscar, Inserm; 2 University Paris Diderot, Paris; ${ }^{3}$ Ensiacet, CIRIMAT, Inpt-Ups-CNRS, Toulouse; ${ }^{4}$ Pôle locomoteur, Service de Rhumatologie, centre Viggo Petersen, AP-HP, Hôpital Lariboisière, Paris, France

Background: Calcium pyrophosphate (CPP) crystal-induced inflammation is mainly driven by interleukin (IL)- $1 \beta$. IL- $1 \beta$ production involves a two-step process including the formation of pro-IL-1 $\beta$ through NF- $\kappa B$ activation and its maturation inti active IL-1 $\beta$ through NLRP3 inflammasome activation. Two CPP crystal phases, namely monoclinic and triclinic CPP dehydrated ( $m$ - and t-CPPD) crystals are identified in synovial fluids. We have recently demonstrated that $\mathrm{m}$ - and $\mathrm{t}-\mathrm{CPPD}$ display different inflammatory potentials marked by a differential level of IL-1 $\beta$ production and other pro-inflammatory mediator expression.

Objectives: We aimed to assess the intracellular pathways upstream CPP crystalinduced IL-1 $\beta$ production, according to the different inflammatory properties of CPP crystal phases.

Methods: Four synthesized and pyrogen-free CPP crystals [a-CPP (amorphous), m-CPPD or tetrahydrated (m-CPPT) and t-CPPD] (Gras P et al. 2014) and monosodium urate (MSU) crystals were used, in vitro, to stimulate THP-1 cell line or mouse bone marrow-derived macrophages (BMDM) in the presence or not of pharmacological inhibitors: Bay-117085 (Bay, NF-кB inhibitor); SB203580 (SB, MAPK p38 inhibitor); SP600125 (SP, JNK inhibitor) and PD98059 (PD, p42/44 MAPK inhibitor). The expression of pro- and anti-inflammatory cytokine genes was assessed by qRT-PCR, and IL- $1 \beta$ or IL- 8 production by ELISA; MAPK phosphorylation by immunoblot. NF- $\mathrm{B}$ B activation was quantified in THP-1 cells containing a gene reporter plasmid under control of NF-KB transcriptional factor. In vivo, we used murine air pouch model to assess the effects of NF-kB inhibition in CPP crystal-mediated inflammation.

Results: We previously demonstrated that in vitro m-CPPD crystals were the most inflammatory CPP crystals and induced a higher production of mature IL-1b associated to a higher expression of NLRP3 protein, and a higher IL-8 production and expression of inflammatory cytokine genes (IL-1 $\beta, I L-6, I L-8, I L-33, T N F$, COX-2) than t-CPPD, m-CPPT and MSU crystals. a-CPP crystals did not have inflammatory property. m-CPPD crystals induced a higher NF-kB activation and a higher phosphorylation p38, p42/44 and JNK MAPKs than t-CPPD and MSU crystals. In parallel, we showed that inhibition of NF- $\mathrm{KB}$ completely abrogated mature IL- $1 \mathrm{~b}$ and IL-8 secretion and pro-IL-1 $\beta$ synthesis induced by all CPP crystals. Inhibition of JNK and p42/44 MAPK with SP and PD respectively, decreased both IL-1 $\beta$ secretion and NF-KB activation induced by CPP crystals. In vivo $\mathrm{IL}-1 \beta$ and $\mathrm{IL}-8$ production and neutrophil infiltration induced by $\mathrm{m}-\mathrm{CPPD}$ crystals were dramatically decreased by NF-кB inhibitor.

Conclusions: Our results showed that IL-1 $\beta$ and IL- 8 production induced by CPP crystals is completely dependent of NF-kB signaling. They suggested that the inflammatory potential of different CPP crystals relied on their capacity to activate both MAPK and NF-кB pathways. Differential level of NF-кB activation is partially dependent of MAPK phosphorylation. Additional studies are ongoing to investigate the underlying mechanisms.

References:

[1] Gras $P$ et al Structure of the calcium pyrophosphate monohydrate phase (Ca2P2O7.H2O): towards understanding the dehydration process in calcium pyrophosphate hydrates. Acta Crystallogr C Struct Chem. 2014.

Disclosure of Interest: None declared

DOI: 10.1136/annrheumdis-2017-eular.4741

\section{THU0035 CYTOKINE PROFILING OF AQUEOUS HUMOR IN BEHÇET'S} DISEASE PATIENTS WITH ACTIVE OCULAR INVOLVEMENT

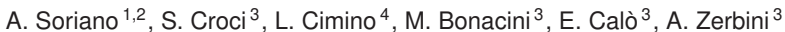
M. Parmeggiani ${ }^{3}$, L. Fontana ${ }^{4}$, C. Salvarani ${ }^{5} .{ }^{1}$ Department of Internal Medicine, Rheumatology Unit, Arcispedale Santa Maria Nuova - IRCCS, Reggio Emilia, Italy, Reggio Emilia; ${ }^{2}$ Campus Bio-Medico University of Rome, Rome; ${ }^{3}$ Clinical Immunology, Allergy and Advanced Biotechnologies Unit; ${ }^{4}$ Ocular Immunology Unit; ${ }^{5}$ Department of Internal Medicine, Rheumatology Unit, Arcispedale Santa Maria Nuova - IRCCS, Reggio Emilia, Italy

Background: Behçet's disease (BD) is a systemic inflammatory disorder with unknown etiology. Uveitis and retinal vasculitis occur in $60-80 \%$ of patients during the disease course. Previous data on cytokine profiling in aqueous humor $(\mathrm{AH})$ of patients with active BD-related uveitis suggested that both T helper (Th) 1 and Th17 cells are involved in immunopathogenesis [1]. Furthermore, higher levels of Natural Killer T (NKT) cells (CD3+ CD56+) have previously been found in $\mathrm{AH}$ of patients with BD-related uveitis as compared to other types of uveitis [2]. However, data on magnitude and patterns of cytokines in BD-related uveitis remain scarce. Objectives: To examine cytokine production as well as frequency of NK and NKT cells in $\mathrm{AH}$ from $\mathrm{BD}$ patients with active uveitis as compared to patients with active Vogt-Koyanagi-Harada (VKH) disease-related uveitis.

Methods: AH from 8 adult patients with BD (1990 ISGB criteria [3]) and active uveitis, and from 7 patients with active VKH were analyzed. Definition of "active" uveitis included $\geq 2$ cells in the anterior chamber (Hogan scale, 1950) and/or 2+ vitritis (Nussenblatt scale, 1990), papillitis, macular edema and retinal vasculitis with active "photo fundus". AH from 5 subjects undergoing cataract surgery were included as controls. Cytokines' concentrations were determined with Bio-Plex Pro-Human cytokine 27-plex assay. Frequency of NK and NKT cells was determined by flow cytometry using anti-CD3, -CD56, -CD16 antibodies. Results: Higher levels of IL-1b, IL-1RA, IL-5, IL-7, IL-6, G-CSF, IFN-g, IP-10, TNF-a IL-8 and MIP1a were found in $\mathrm{AH}$ from patients with $\mathrm{BD}$ and VKH compared to controls. G-CSF and IFN-g were detected in AH from BD and VKH patients, but not in the control group. Lower levels of GM-CSF were found in BD and VKH patients, while IL-6 levels were 3000 -fold increased, as compared to controls. No differences were detected between BD and VKH patients in terms of cytokine levels. However, $\mathrm{AH}$ from $4 \mathrm{BD}$ patients showed a peculiar distinct cytokine pattern, when analyzed by unsupervised cluster analysis. Intriguingly, a higher amount of neutrophils $/ \mathrm{mL}$ was found in $\mathrm{AH}$ from these $4 \mathrm{BD}$ cases. Frequency of NKT (CD3+ CD56+) cells was higher in BD patients as compared to $\mathrm{VKH}$, while that of $\mathrm{NK}\left(\mathrm{CD} 56+\mathrm{CD}^{\text {neg }}\right)$ cells was similar and that of $\mathrm{T}$ cells $\left(C D 56^{\text {neg }} C D 3+\right)$ was lower. Finally, no difference was found between NK subsets in terms of proportion of CD16+ cells in both BD and VKH groups.

Conclusions: $\mathrm{AH}$ of both $\mathrm{BD}$ and $\mathrm{VKH}$ groups showed increased levels of IL-6, G-CSF and IFN-g, which might suggest their potential role in the immunopathogenesis of these types of endogenous uveitis. However, a distinct cytokine profile able to distinguish the two conditions remains to be identified. Additionally, our preliminary results confirm the previous observation of increased NKT cells levels in BD uveitis as compared to VKH.

References:

[1] El Asrar A.M., et al. Clin Immunol 2011;139:177-84

[2] Yu H.G., et al. Clin Exp Immunol 2004;137:437-443.

[3] International Study Group for Behçet's Disease. Lancet 1990;335:1078-80.

Disclosure of Interest: None declared

DOI: 10.1136/annrheumdis-2017-eular.6635

\section{THU0036 ROLES OF IL-8 IN RANK-L- AND ACPA-MEDIATED OSTEOCLASTOGENESIS}

A. Cîrciumaru, Y. Liu, A. Krishnamurthy, S.M. Mia, V. Joshua, M. Engström, V. Malmström, M. Sun, H. Wähämaa, B. Rethi, A. Catrina. Karolinska Institute, Karolinska University Hospital, Stockholm, Sweden, Stockholm, Sweden

Background: We have shown previously in various experimental models that anti-citrullinated protein antibodies (ACPAs) can contribute to bone erosion and arthralgia through IL-8 dependent mechanisms.

Objectives: In cell cultures osteoclasts (OCs) played a prominent role in the ACPA-induced IL-8 secretion and therefore we decided to characterize in detail the autocrine regulation of $\mathrm{OC}$ differentiation by IL-8 in the presence or absence of ACPAs.

Methods: Peripheral blood CD14-positive monocytes were used to generate OCs in the presence of M-CSF and RANKL. Expression levels of IL-8 and CXCR1/2 were monitored during OCs maturation using intracellular flow cytometry, cytometric bead array, immunohistochemistry and RT-PCR. Inhibition of IL-8 and its receptors were performed in OC cultures using IL-8 neutralizing antibodies and small molecule CXCR1 and CXCR2 antagonists (Reparixin, SB-332235), in the presence or absence of polyclonal ACPAs. OC numbers were counted using light microscope after TRAP staining.

Results: Macrophages and developing OCs secreted high levels of IL-8 in response to the cytokine M-CSF. IL-8 expression kinetic was characterized by transient peaks when new cytokine was added, followed by very low level of steady-state production. In the presence of ACPAs IL-8 production increased 3-4 fold during late phases of OC differentiation. With the help of IL-8 neutralizing antibodies or inhibitors acting on the IL- 8 binding chemokine receptors CXCR1 and CXCR2 we showed that the M-CSF induced IL-8 plays a crucial role in OC differentiation in the presence of exogenous RANK-L. In the presence of ACPAs OC differentiation was further enhanced in response to the ACPA-mediated increase in IL-8 production. The CXCR1 and CXCR2 inhibitors Reparixin and SB332235 significantly inhibited the RANKL-mediated osteoclastogenesis at $100 \mu \mathrm{M}$ and $10 \mu \mathrm{M}$ respectively, both in the presence or absence of ACPAs. By using the inhibitors at low concentrations we could counteract the ACPA-mediated increase in OC development without interfering with steady state OC differentiation.

Conclusions: Our findings further support an important role for IL-8 in bone biology and in the ACPA-mediated OC differentiation both under physiological conditions and in the presence of ACPAs. Together with previous data showing in vivo, our data indicated that small molecule CXCR1 and CXCR2 antagonists could provide novel therapeutic tools for targeting OCs in ACPA+ individuals.

Disclosure of Interest: None declared DOI: 10.1136/annrheumdis-2017-eular.6886 